\title{
Examination of Underlying Factors in Success of TikTok
}

\author{
Mingyin $\mathrm{Yao}^{* 1}$ \\ ${ }^{1}$ Xi' an Jiaotong Liverpool University \\ ${ }^{*}$ Corresponding author. Email: mingyinyao23@sina.com
}

\begin{abstract}
This paper firstly performed a customer profile analysis by introducing seven assumptions related to TikTok customers, through researching, several conclusions can be reached: the majority of users are under 25 years old. TikTok users want an easy access to pass the time, save content and, more importantly, get personalized recommendations from the algorithm. Secondly, the revenue model is explained. Research shows that TikTok makes money in three main ways: advertising (both on the front page and in the feed), event marketing, and livestreaming. Finally, a business model canvas is made based on the utilization, easy to make friends, mass material copyright, technical support, partner market, consumer end and cost and other aspects.
\end{abstract}

Keywords: TikTok, factors of success, Customer profile, Revenue model, Business canvas

\section{INTRODUCTION}

The increasing reliance on mobile devices and the rapid updating on the network technology make mobile videos available and become an everyday possibility. Studies of consuming videos on mobile devices suggested that people prefer to view short episodes on their devices when accessing mobile videos, which may become a factor of the introduction of TIkTok [7]. TikTok, also known as Douyin in China, was launch by a Chinese technology company called ByteDance in 2016 [1]. It is a new social network site that enable users to watch, create, edit and share short-form video clips with 15 seconds in length. There are rich content formats consisting of filters and BGM (the short of 'background music'), to some extent contributing to the global viral phenomenon of TikTok usage and this short video sharing platform is claimed to become one of the fast-growing applications all over the world [2]. Data collected by Statista suggests that there are on average 800 million active users on TikTok worldwide by the year 2020 and TikTok has been reported to be the world most frequently first install application in 2018 [13]. TikTok seems to have been getting successful in the market, but how? The aim of this paper is to examine the reasons why TikTok is able to capture quite a significant share of the market by illustrating three methods (customer profiling, revenue model and business model canvas, respectively).

\section{BUSINESS}

\subsection{Customer Profiling}

It is of great important to profile the customer when setting a company. In order to define the customer of TikTok, several hypotheses have been made aiming at different aspects based on literature research and, including the gender and age of the customer, their pains as well as gains.

\#1 Gender Hypothesis: There are more female TikTok users than male TikTok users.

\#2 Age Hypothesis: the adolescence group, specifically the mid-adolescence (15-17) and the late adolescence (18 to 24 ), is the main customer in TikTok usage (The age range of mid-adolescence and late adolescence is defined by Psychology Today [12].)

\#3 Gain Creator Hypothesis: Swiping through TikTok is a good way for customer to kill time.

\#4 Gain Creator Hypothesis: The archiving character of TikTok makes it convenient for users to download and save their interesting and creative videos.

\#5 Gain Creator Hypothesis: The artificial intelligence algorithms provides personalized video recommendation to every user which attracts the interest of customers. 
\#6 Pain Reliver Hypothesis: Uploaders' willing of expressing themselves is satisfied through using TikTok.

\#7 Pain Reliver Hypothesis: the design of TikTok make it possible for every user to become famous if the video produced is attractive and gets lots of Likes and Views, which satisfies users need of fame-seeking and social recognition.

In order to examine the above hypotheses, relevant literature has been examined regarding the above hypothesis and summarized as follow.

The TikTok users' gender ratio is analyzed [10]. It led to a result that the percentage of male TikTok users is $53 \%$ and resulting to that of female TikTok users is $47 \%$, while it is said that by the end of the first quarter of the year 2020, the man users in Douyin which is the Chinese original version of TikTok has the proportion of $43 \%$ and woman users using Douyin counts for $57 \%$ of the total Douyin users [5]. With regard to the users of TikTok, the hypothesis that the number of female users outweighs that of male users seems to be wrong. Moreover, if consider the users by gender globally, the gender hypothesis could not be proved as well, due to the lack of statistics.

GlobalWebIndex, a web market research company illustrated in its study that in 2019, 40 percent of TikTok users are made up of young generations aging between 16 and 24 that is in the range of mid-adolescence and late adolescence [6]. In particular, in order to give a further demonstration of this age hypothesis, four countries were selected, and their user age ranges were analyzed. First, we collected and ranked the TikTok (Douyin) usage data of people in each country from Statista which is an authoritative statistical website, and then plotted the top four bar images.

\section{Top 4 Countries with the Largest TikTok}

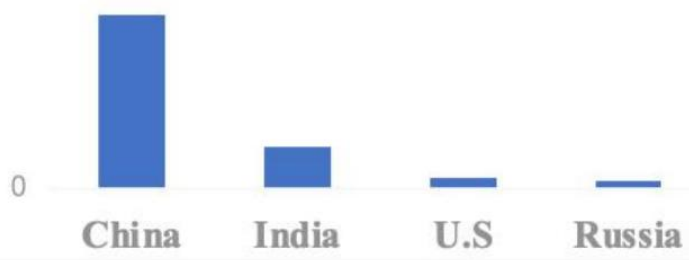

Figure 1. Top 4 Countries with the Largest TikTok Users

It can be illustrated from the above bar chart that the countries with the largest number of TikTok (Douyin) users is China (699.36 million), followed by India (160.12 million), U.S. (41.65 million) and Russia (28.16 million). It is stated that approximately sixty percent of the Douyin streaming users in China are under 30 years old [14]. In the United State, 32.5 percent of the total TikTok uses lies in the age group from 10 to 19 , followed by a 29.5 percent of users aging between 20 and 29 [15]. Besides, study shows that the majority of Tiktok users in Russia were aged from 13 to 17 years old, which represents a proportion of 43 of the total users on the application [1]. Surprisingly, only 3 percent of total accounts were 35 years old or above.

With regard to the three gain creator hypotheses, It is argued in his study that the widely used algorithm contributes to the satisfaction of each TikTok user' $\mathrm{s}$ personal preference about mobile video, which makes TikTok an entire interest-driven (both what you like and what you dislike) application [3]. TikTok has an extreme ability of customization. To be more specific, the For You Feed will deliver you series of video which are presumed that are likely to suit your interest, which is supported by a recommend system powered by strong algorithm. In particular, every one of the For You Feed is not exactly the same whilst there may exist a situation that some users may encounter same trended videos, this Feed is basically unique for every user [16]. The TikTok platform studies from every interaction happened during the usage, including the times and duration of you watching a particular video, the videos that are lying in your Favorites, seen as Figure 2 (Long press to add the videos that are interests you in the Favorites so that you can easily find them and review them again.) and the videos that you press a button and click on 'not interest' (Figure 3).

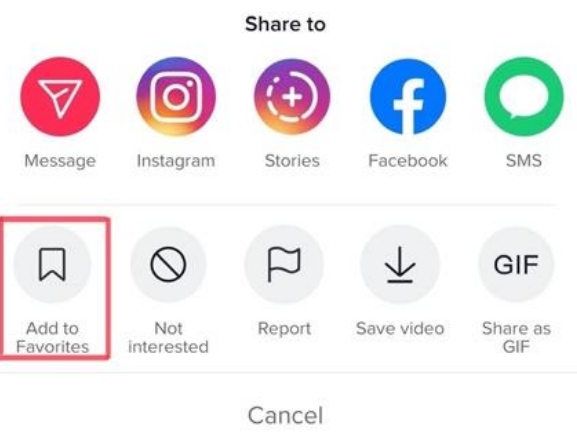

Figure 2 Favorite Button in TikTok 


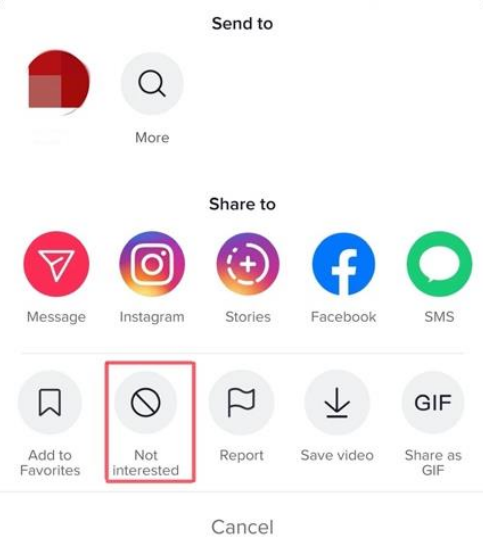

Figure 3 Not Interest Button in TikTok

Thus, people easily get into the customized feed and it is a good way to kill time. Moreover, the archiving character (Figure 4) makes users save their creative video into their phone conveniently in order to save and share wonderful moments and their own creativity.

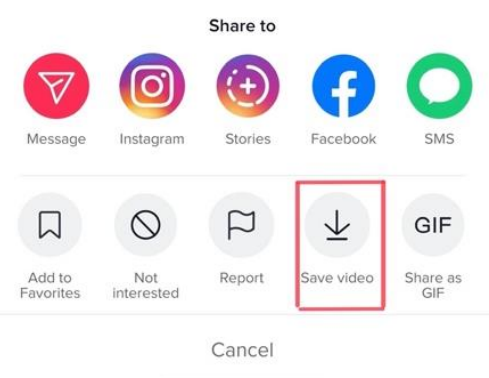

Figure 4 Archiving Character in TikTok

Regarding to the two pain reliver hypotheses, it is illustrated that adolescence has a non-negligible level of desire to express themselves. Therefore, self-expression is of vital importance in youth' $s$ development of mental health. TikTok provide teens, the largest group among all users, a platform to express themselves, through the user-based system. To give further explanation, users interaction is a fundamental way for TikTok users to express themselves. The video users like, or share gives out the information of your preference while the comments you post and content you create are the reflections of your thoughts. Besides, two of the most significant predictors to TikTok usage behavior are users' motivations which includes social recognition and the pursuit of fame [11]. With the assistance of TikTok' $\mathrm{s}$ recommendation system, especially the push-flow mechanism, any content uploader who produces sophisticated, trendsetting or thought-provoking videos can acquire an opportunity to gain quite amount of likes and followers. Based on the algorithm, once a user likes you video, their subsequent outputs will frequently appear on his or hers Feed. If a user continues to make popular content, his or hers fanbase will continue to surge, which meets the need of fame-seeking and social recognition.

\subsection{Revenue Model}

It may be confused for some people that how TikTok makes money, in this part, three arguments are examined in the following paragraphs in order to analyze its revenue model.

\subsubsection{Advertising Revenue}

First of all, advertising revenue is a majority source in the revenue structure of TikTok. advertisement can be divided into homepage advertisement and in-feed advertisement. When it comes to the homepage advertisement, a wild spread form of mobile phone apps, the most common way is to display ads for approximately 3 seconds when the users open the apps. Advertising fee in this form is based on daily active users and opening frequency of a particular app, which has a usual charging mode of cost per time [4]. The price seems to show difference between static picture and dynamic video, according to the data in 2018, the dynamic ads cost 85,000 USD more than static ads with the price of 485,000USD per day. Nowadays, TikTok gradually becomes the trend of the age, with the popularity of being used in more than 154 countries/areas, and the rapid growth in the number of users in recent years which already achieved more than 800 million (exceeding Reddit, Snapchat and LinkedIn). Advertisement effect and fee is increasing simultaneously bases on the facts above. As for in-feed advertisement, they are similar to the ads we see while rolling in the Instagram. When users scroll through the For You Feed on the TikTok, ads in the Feed are displayed which can last between 9 and 15 seconds. Unlike traditional ads, in-feed ads are displayed more like banners or very short videos inserting in relatively larger videos. This method helps to better combine advertisements with normal content, so that it can reduce users' aversion. The charging mode of the platform is usually CPM which is the price of advertisement viewed 100 times, and cost about 50USD per CPM accordingly [9]. With the support of artificial intelligence technology, TikTok can currently be charged adaptively by monitoring the data of every advertisement, including number of comments, sharing, viewers and the residence time of users. According to the AI technology and big data, TikTok can later recommend ads that are more suitable for users, thus attracting users to consume. In this case, the manufacturer will be willing to invest more advertising costs, which helps TikTok create the first closed-loop ecosystem.

\subsubsection{Event marketing revenue}

Event marketing is also a way for TikTok to make money. According to the big data, the platform will publish events to the users. Participants are 
unconsciously influenced by the event and may start to recognize or have a better impression of a particular brand. These events are often associated with the entertainment industry or food industry. For instance, for music release, musicians can buy a special event from the platform, asking users to use their new songs as background music for their video, so that the popularity of new songs will increase rapidly. Compared with the traditional event marketing, the utilization of TikTok for event marketing makes buyer avoid complicated preparatory work and the way of publishing and thus it becomes one of the ways to make profit.

\subsubsection{Livestream revenue}

The third point is a kind of revenue weighs in small quality but forms in huge quantities. In live rooms of many APPs, numbers of viewers are used to reward the anchors by buying virtual gifts. For live streaming companies, the virtual gift commission has been proved to be a reliable monetization method. The debut of Live Streaming of TikTok showed in November 2017, and soon TikTok began generating revenue through virtual gift commission. In the live room, you can exchange $\$ 1.29$ for 100 TikTok-coin, \$6.49 for 500 TikTok-coin, $\$ 26.99$ for 1000 TikTok-coin, $\$ 1.29$ for 200 TikTok-coin, $\$ 66.99$ for 5000 TikTok-coin, \$134.99 for 10000 TikTok-coin respectively, and the prices range from 1 coin to 1000 coins. When anchors receive virtual gifts, TikTok will distribute the revenue with anchors in particular proportion to make real profit.

\subsection{Business Canvas Model}

Combining the above customer profiling and revenue model with the existing information, the following business canvas model is made.

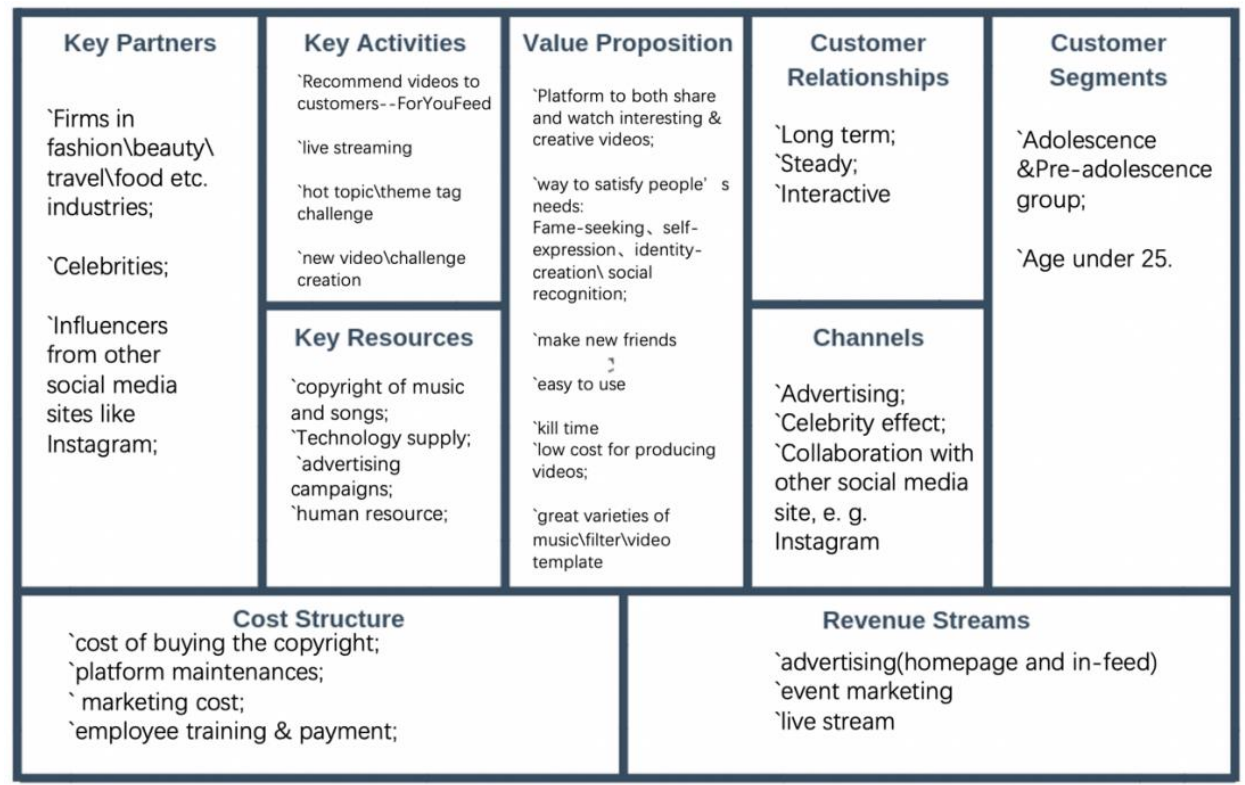

Figure 5 Figure Business Canvas Model of TikTok

To start with, the value proposition includes 7 elements. Firstly, TikTok is an easy to utilize platform providing users a place to get the sense of happiness from interesting video contents with very low cost for users to create and enjoy what they needed. For instance, every user can freely watch the video they like after registration physically and economically. Furthermore, if the users incline to spread their video to others, they can pay small amount of money to achieve their goals.

Beyond entertaining the users, tiktok also offers an incredibly excellent platform which synthesizes mostly all advantages of popular social networks. Through pressing 'LIKE' button or leaving comments, TikTok seems to provide users a channel to make new friends and even to broader their social network.
Except what we discuss above, because of owning huge intellectual property rights of music. TikTok has great varieties of music and filter template and thus creators can have a wide range of choices when making a trendsetting video.

As for the technical support, the recommend system which equipped with powerful algorithm provide an opportunity to satisfy three needs of their users (fame-seeking, self-expression and social recognition, respectively). TikTok's key activities includes not only live streaming and video $\backslash$ challenge creation, but also a customized recommend system 'For You Feed' and hot topic tag challenge. The key resources are partly made up of the copy right of music and song, the other parts are technology supply like algorithm, advertising campaigns and human resources. 
When we talk about the business canvas mode, partners are always essential. As for tiktok, it has an abrest of time view for its partners. Under this very version, fashion, beauty or travel and food industries and so on become one of them. Besides, TikTok puts celebrities and influencers from other social media sites into its partner list.

When it comes to the deeper side, consumer, TikTok aims to build a long term, steady and interactive relationship with their customers. The comments and suggestions on the various functions of TikTok is are welcomes. This application is open for general public, especially mid adolescence and late adolescence (age between 15 and 24) while the gender ratio is not likely to be determined. TikTok basically get attach to new customer by advertising, collaborate with celebrities and also, collaborate with other social media site. For instance, Instagram [8].

The cost is structured by platform maintenances, marketing cost and human resources cost, including employee training and payment fee. Apart from those, cost of buying the copyright of music and songs is also a main cost for TikTok. To construct the revenue model, both homepage advertising and in-feed advertising are the most significant monetization method of TikTok. Moreover, TikTok also makes money from event marketing and the share of live stream reward.

\section{CONCLUSION}

Three main parts are discussed in this paper. Firstly, customer profiling is examined by introducing 7 hypotheses related to TikTok's customers and researching to prove or oppose them. At the end of this section, we can conclude that most of the users are aging under twenty-five. People using TikTok are expecting to kill time, save their content conveniently and furthermore, to receive personalized recommendation from algorithm. Besides, the revenue stream is also illustrated. Research shows that there are three main monetization methods, advertising (both homepage and in-feed advertisement), event marketing and live streaming reward. Finally, based on that, a business model canvas is made in terms of utilization, easily accessible to make friends, huge copyright of materials, technical support, partners market, consumer side and costs.

\section{ACKNOWLEDGEMENT}

First and foremost, I would like to show my deepest gratitude to my teachers and professors in my university, who have provided me with valuable guidance in every stage of the writing of the examination of underlying factors in success of TikTok. Besides, I would also like to express my gratitude to my family and friends. It is their support that provides me the motivation to persist in finishing this paper.

\section{REFERENCES}

[1] Alagina, D. (2021), Share of TikTok users in Russia in 2020, by age group. Available at: https://www.statista.com/statistics/1101357/russiatiktok-users-age/ (Accessed: 28 February 2021)

[2] Bereznak, A. (2019), 'Memes are the new pop stars: how TikTok became the future of the music industry', The Ringer. Available at: www.theringer.com/tech/2019/6/27/18760004/Tik Tok-old-town-road- memes-music-industry (Accessed: 28 February 2021)

[3] Cuofana, G. (2020), TikTok Business Model: The Rise of Creative Social Media Powered By AI. Available at: https://fourweekmba.com/tiktok-business-model/\# How_does_TikTok_make_money (Accessed: 28 February 2021)

[4] Gu, E., He, S., Wu, J., Study on the Evolution Mechanism of Social Media Business Model based on Value Co-creation Theory: Taking Short Video Social Media TikTok as an Example. doi: 10.26914/c.cnkihy.2020.016381

[5] Graziani, T. (2020), Douyin, Kuaishou, Red, Bilibili: Where to Promote Your Brand in China Besides WeChat? Available at: https://walkthechat.com/douyin-kuaishou-red-bilib ili-where-to-promote-your-brand-in-china-besideswechat/ (Accessed: 28 February 2021)

[6] GlobalWebIndex, https://www.globalwebindex.com (Accessed: 28 February 2021)

[7] Hara, K. O., Mitchell, A. S. and Vorbau, A. (2007) 'Consuming Video on Mobile Devices', SIGCHI Conference on Human Factors in Computing Systems, pp. 857-866. doi: $10.1145 / 1240624.1240754$

[8] Haenlein, M., Anadol, E., Farnsworth, T., Hugo, H., Hunichen, J. and Welte, D. (2020), Navigating the New Era of Influencer Marketing: How to be Successful on Instagram, TikTok, \& Co. doi: 10.1177/0008125620958166

[9] Jiang, X. (2019), 'Research on TikTok APP Based on User-Centric Theory', Applied Science and Innovative Research, p.28. doi: 10.22158/asir.v3n1p28

[10] Kemp, S. (2020), Digital 2020: October Global Statshot. Available 
https://datareportal.com/reports/digital-2020-octob er-global-statshot (Accessed: 28 February 2021)

[11] Omar, B. and Dequan, W. (2020), "Watch, share or create: the influence of personality traits and user motivation on TikTok mobile video usage", International Journal of Interactive Mobile Technologies (Ijim), Vol. 14 No. 4, pp. 121-137. Available at: https://www.researchgate.net/publication/3398861 41_Watch_Share_or_Create_The_Influence_of_Pe rsonality_Traits_and_User_Motivation_on_TikTo k_Mobile_Video_Usage (Accessed: 28 February 2021)

[12] Psychology Today, https://www.psychologytoday.com/us/basics/adole scence (Accessed: 28 February 2021)

[13] Statista, https://www.statista.com (Accessed: 28 February 2021)

[14] Thomala, L. L. (2020), Douyin (TikTok) streaming user age distribution in China 2020. Available at: https://www.statista.com/statistics/1132642/chinadouyin-streaming-user-age-distribution/ (Accessed: 28 February 2021)

[15] Tankovska, H. (2021), TikTok user ratio in the U.S. 2020, by age group. Available at: https://www.statista.com/statistics/1095186/tiktokus-users-age/ (Accessed: 28 February 2021)

[16] Zhao, Z. (2021), 'Analysis on the "Douyin (Tiktok) Mania" Phenomenon Based on Recommendation Algorithms, Web of Conferences, Vol 235, p.03029. doi: 10.1051/e3sconf/202123503029 